\title{
Environment map generation in forest using field robot
}

\author{
Noboru Takegami \\ Department of Mechanical Information Science and Technology, Kyushu Institute of Technology \\ 680-4, Kawazu, Iizuka-City, Fukuoka, 820-8502, Japan \\ Eiji Hayashi \\ Department of Mechanical Information Science and Technology, Kyushu Institute of Technology \\ 680-4, Kawazu, Iizuka-City, Fukuoka, 820-8502, Japan \\ Ryusuke Fujisawa \\ Department of Mechanical Information Science and Technology, Kyushu Institute of Technology \\ 680-4, Kawazu, Iizuka-City, Fukuoka, 820-8502, Japan \\ E-mail: takegami@mmcs.mse.kyutech.ac.jp, haya@.mse.kyutech.ac.jp,fujisawa@.ces.kyutech.ac.jp \\ http://www.kyutech.ac.jp/
}

\begin{abstract}
The employment rate of young people in Japanese forestry tends to decline, and the unmanaged forest area is expected to increase in the future. Therefore, in our laboratory we propose an autonomous field robot with all terrain vehicles that focuses on the automation of work. The robot automates weeding and observation in the forest. In this research, we observed trees by generating an environmental map in the forest using Simultaneous Localization and Mapping (SLAM). The error of the generated environmental map was about $1[\mathrm{~m}]$.

Keywords: Field Robot, ROS, SLAM, Mapping, Forestry
\end{abstract}

\section{Introduction}

In Japan's forestry industry, the decline in timber prices and labor shortages are causing the decline of forestry. However, much labor and time have to be spent on forest management and conservation. Especially, In-forest investigations and weeding work are burdensome for forestry workers. The in-forest investigations measures and manages the amount of resources, the amount of growth and quality of trees. The weeding work removes weed that impede the growth of seedlings after afforestation. In order to reduce the work burden, mechanization and automation of forestry work are required. In this research, we developed an autonomous moving robot with platform of all-terrain vehicle with high moving ability in rough terrain. With this robot, we aim to automate the weeding work and resource management within the forest. We introduced the Simultaneous Localization and Mapping (SLAM) system that performs self-position estimation and environmental map generation based on Robot Operating System (ROS) to the robot. ${ }^{1}$

\section{The Robot Platform}

Fig.1 shows the robot developed in this study. We will operate the robot on a steep slope in the actual mountain hillside, and the road is rough. Thus, the robot is based on an ATV (Kawasaki, Inc.) to handle on the rough terrain. The size of robot is following; length: 1400 [mm], width: 900 [mm], height: 1200 [mm]. In the Japanese

(C) The 2020 International Conference on Artificial Life and Robotics (ICAROB2020), Jan. 13-16, B-Con Plaza, Beppu, Oita, Japan 
forestry work, the planting interval is approximately 2.5 $[\mathrm{m}]$, which is narrow compared to forestry regulations in other countries. The size of our developed robot is sufficiently small compared to the planting interval that it can easily travel into the native forest. The robot has four wheels, the front two wheels are turning and the rear two wheels are driving. The external environment is recognized by RealSense R200 (Intel, Inc.) which is constructed with a depth sensor and a RGB-CCD camera, and equipped on the ATV. The depth sensor can acquire an object's position as three-dimensional (3D) point cloud data via an infrared laser measurement. The maximum measurable depth direction of the sensor is 15 [m]. In addition, the robot has an inertial measurement unit (IMU) for pose detection and RTK-GPS for global localization. The power source is gasoline engine. The brake lever, accelerator lever, and steering are all controlled by DC motors. A weeding task is performed by a weeding mechanism that has multi blades and attached on the front of the ATV.

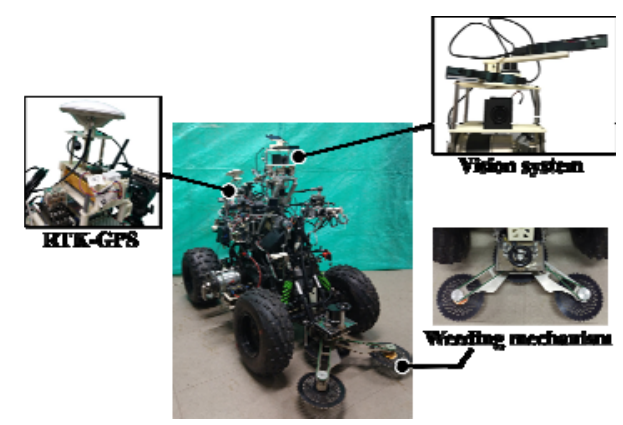

Fig. 1. Outline of robot appearance

\section{Map Generation System}

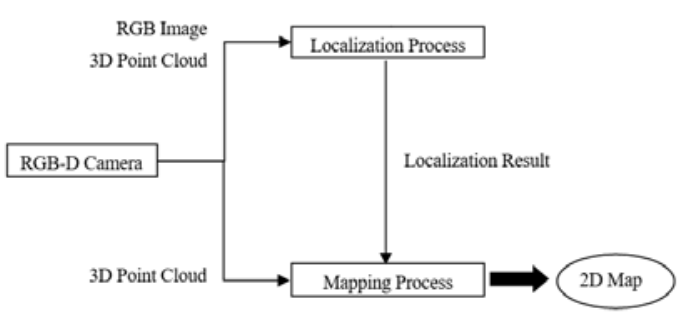

Fig. 2. Overview of the system

Fig.2 shows the overview of the environmental map generation system introduced on this robot. This system configures SLAM using ROS. This system is divided into the two processes, localization process and mapping process.

\subsection{Localization process}

In localization process, RGB image and $3 \mathrm{D}$ point cloud data obtained from RealSense R200 are used as input. We used ORB-SLAM2 for localization process. ${ }^{2}$ In ORBSLAM2, the ORB feature point is obtained from the RGB image, and the camera pose is estimated from the matching result of the ORB feature points at discrete times $\mathrm{t}-1$ and $\mathrm{t}$. ORB feature points are robust against violent motion and interference of light. The camera pose is converted to metric units by using a $3 \mathrm{D}$ point cloud data.

As shown in Fig.3 (a), even when the robot moves along the same path, an error occurs in the estimated selfposition. This error causes a problem that environmental map generation does not work well. Therefore, we calculate the similarity of ORB feature points between current and previous RGB images. Then, if there is an RGB image whose similarity is higher than the threshold value, the self-position estimation result between that RGB image and the current RGB image is corrected. The results are shown in Fig.3 (b).

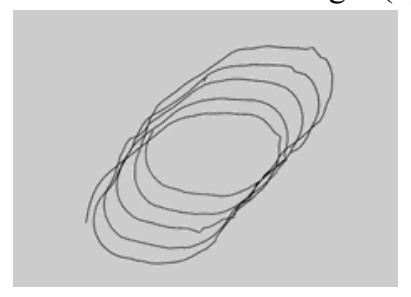

(a) Before correction

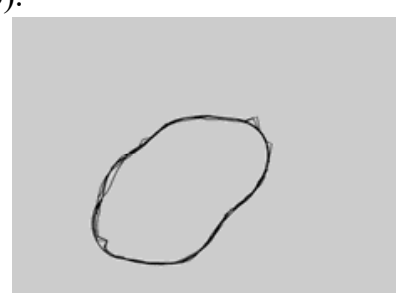

(b) After correction
Fig. 3. Localization result

\subsection{Mapping process}

In mapping process, 3D environmental map and 2D environmental map are generated using localization process results and 3D point cloud.

The $3 \mathrm{D}$ environmental map is generated by arranging $3 \mathrm{D}$ point cloud in space. ${ }^{3}$ If the movement path of the robot is corrected by localization process, the position of the $3 \mathrm{D}$ point cloud is corrected.

When generating a 2D environmental map, identification of the ground and obstacles of the 3D point cloud is performed. The identification is divided into the two steps. First, Estimate the ground using the least squares method for any 20 point clouds and calculate the normal vector. Second, the vertical component of the normal vector is compared with the threshold, and if it is larger than the threshold, the point cloud is identified as the 
ground. The point clouds other than the ground is obstacle. This identification is performed on all point clouds, and the point cloud are projected on a 2D plane to generate a $2 \mathrm{D}$ environmental map. All point clouds, ground and obstacle are shown in Fig.4.

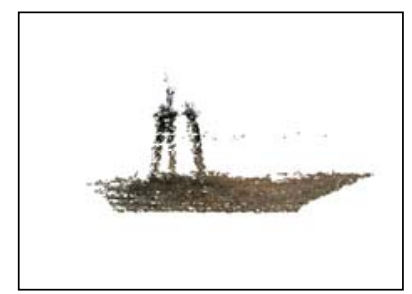

(a) All point clouds

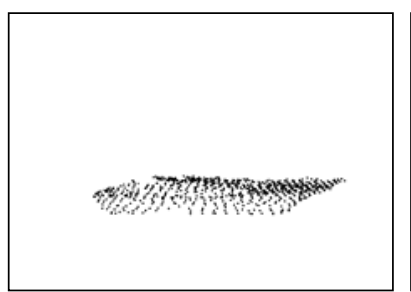

(b) Ground point clouds

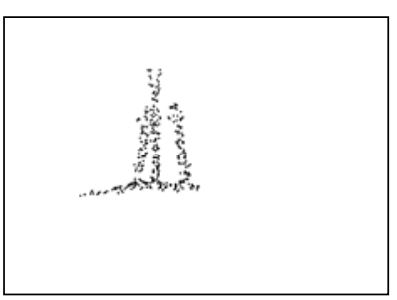

(c) Obstacles point clouds
Fig. 4. Point clouds

\section{Experiment}

We evaluated the constructed system using the robot movement results at the experimental site in our university. The experimental site consists of 39 trees, and there are no objects other than trees. The coordinates of the center of a standing tree are measured in advance using RTK-GPS, and this coordinate is used as a true value. The generated 3D environmental map is shown in Fig.5, and the 2D environmental map is shown in Fig.6. The true value of the tree coordinate, the estimation value of the tree coordinate, and the error is shown in Table 1. A unique number is set for tree. The estimated value is the central coordinates of the clustering result by Euclidean distance. The minimum value of the $x$ coordinate error is $0.04[\mathrm{~m}]$ at No.30, and the maximum value is $-1.8[\mathrm{~m}]$ at No.35. The minimum value of the $y-$ coordinate error is $0.15[\mathrm{~m}]$ at No.8 and the maximum value is $1.95[\mathrm{~m}]$ at No.44. The error of more than $\pm 1.5[\mathrm{~m}]$ occurred at No.35, 39 and 44 .

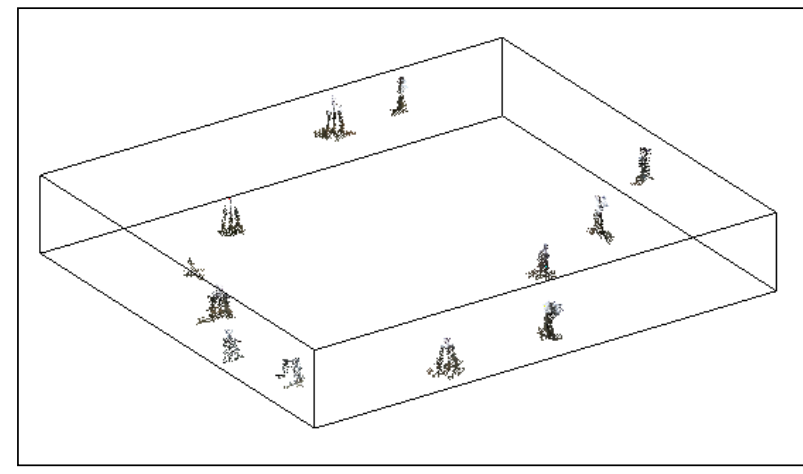

Fig. 5. 3D environmental map

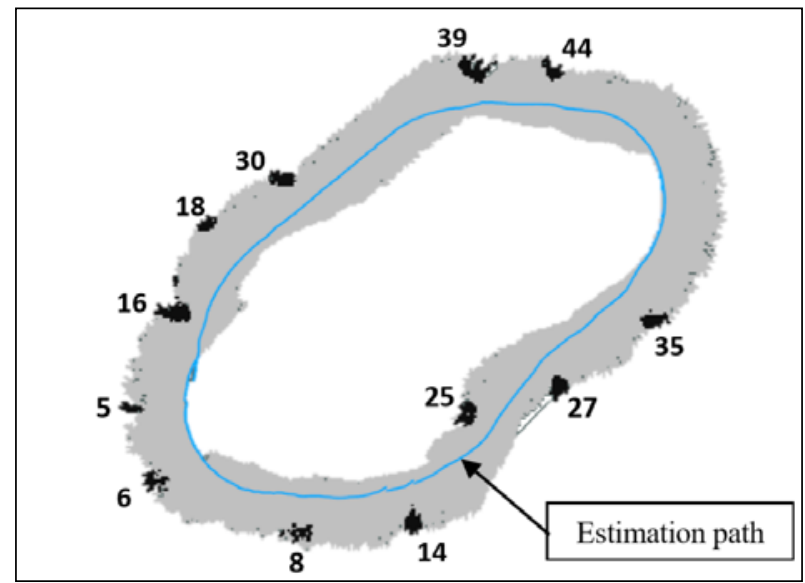

Fig. 6. 2D environmental map

Table 1. True/estimation value and error

\begin{tabular}{|r|r|r|r|r|r|r|}
\hline & \multicolumn{3}{|c|}{ x-coordinate } & \multicolumn{3}{|c|}{-coordinate } \\
\hline No. & True value[m] & estimation value[m] & error[m] & True value[m] & estimation value[m] & error[m] \\
\hline 5 & 0.91 & 0.52 & 0.39 & 2.11 & 3.05 & -0.94 \\
\hline 16 & 0.66 & 0.30 & 0.36 & 6.52 & 5.48 & 1.04 \\
\hline 18 & 0.34 & 0.27 & 0.07 & 10.12 & 9.35 & 0.77 \\
\hline 30 & 2.44 & 2.40 & 0.04 & 12.22 & 12.60 & -0.38 \\
\hline 39 & 7.35 & 8.14 & -0.79 & 18.32 & 20.02 & -1.70 \\
\hline 44 & 10.15 & 11.06 & -0.91 & 19.52 & 21.47 & $-\mathbf{1 . 9 5}$ \\
\hline 35 & 17.85 & 19.72 & -1.87 & 13.02 & 13.97 & -0.95 \\
\hline 27 & 16.25 & 17.26 & -1.01 & 8.82 & 9.83 & -1.01 \\
\hline 25 & 13.35 & 14.23 & -0.88 & 6.12 & 7.17 & -1.05 \\
\hline 14 & 12.45 & 13.47 & -1.02 & 2.00 & 2.17 & -0.17 \\
\hline 8 & 8.55 & 8.90 & -0.35 & 0.13 & 0.28 & -0.15 \\
\hline
\end{tabular}

\section{Consideration}

The error of the coordinates of No.35, 39 and 44 is larger than that of other trees. The reason is that the error of selfposition estimation is large because the movement distance from No.30 to No.39 and No.44 to No.35 are longer than the movement distance between other trees. In the RGB image when moving between No.44 and No. 
35 , although ORB feature points can be obtained from the ground, but there are few trees within the range recognized by RealSense R200, so almost ORB feature points are not obtained from trees. The ORB feature points that can be obtained from the ground are unstable because it is difficult to obtain ORB feature points from the same place at discrete times $t-1$ and $t$. Therefore, it is considered that the matching of ORB feature points can't be performed sufficiently, and the accuracy of selfposition estimation is degraded. When matching ORB feature points, it is considered necessary to track ORB feature points obtained from unstable ground.

\section{Conclusion}

In this study, we constructed SLAM by ROS and generated the environmental map in the forest using robot. It was possible to identify trees and ground even in a site simulating a forest with complex terrain. In addition, it was found that self-position estimation is possible when there is an obstacle. However, it was found that an error occurs when there is no obstacle.

In the future, when matching ORB feature points, not only RGB images at discrete time $\mathrm{t}-1$ and $\mathrm{t}$ are used, but also matching with RGB images before $\mathrm{t}-1$ is used. By maintaining unstable ORB feature points obtained from the ground, we aim to develop a system that reduces the error of self-location estimation. And we aim at the further accuracy improvement of self-position estimation by mutually compensating the self-position obtained from this system and RTK-GPS.

\section{References}

1. M.Quigley, B.Gerkey, K.Conley, J.Faust, T.Foote, J.Leibs, E.Berger, R.Wheeler, A.Y.Ng. "ROS: an open-source Robot Operating System," In Proceedings of the IEEE International Conference on Robotics and Automation, Vol.3, p.5 12-17 May 2009.

2. R. M. Artal and J. D. Tardós. "ORB-SLAM2: an OpenSource SLAM System for Monocular, Stereo and RGB-D Cameras", IEEE Transactions onRobotics, Vol.33, No.5, pp.1255-1262, 2017.

3. M. Labbé and F. Michaud. "RTAB-Map as an OpenSource Lidar and Visual SLAM Library for Large-Scale and Long-Term Online Operation," Journal of Field Robotics, 2018 